\title{
1 Optogenetics reprogramming of planktonic cells for biofilm
}

\section{2 formation}

3 Aiguo Xia ${ }^{\mathrm{a}, 1}$, Shuai Yang ${ }^{\mathrm{a}, 1}$, Yajia Huang ${ }^{\mathrm{b}, 1}$, Zhenyu Jin ${ }^{1}$, Lei Ni ${ }^{1}$, Lu Pu ${ }^{1}$, Guang Yang ${ }^{\mathrm{b}, 2}$ and Fan

$4 \quad \operatorname{Jin}^{\mathrm{a}, \mathrm{c}, \mathrm{d}, 2}$

$5 \quad{ }^{a}$ Hefei National Laboratory for Physical Sciences at the Microscale, University of Science and

6 Technology of China, Hefei 230026, P. R. China

7 bepartment of Biomedical Engineering, College of Life Science and Technology, Huazhong

8 University of Science and Technology, Wuhan 430074, P. R. China

$9 \quad{ }^{\mathrm{c}}$ Department of Polymer Science and Engineering, University of Science and Technology of

10 China, Hefei 230026, P. R. China

$11{ }^{\mathrm{d}}$ CAS Key Laboratory of Soft Matter Chemistry, University of Science and Technology of

12 China, Hefei 230026, P. R. China

$13 \quad{ }^{1}$ A.G.X, S.Y. and Y.J.H. contributed equally to this work.

$14{ }^{2}$ To whom correspondence should be addressed:

15 E-mail: fjinustc@ustc.edu.cn; yang_sunny@yahoo.com. 


\section{Abstract}

17 Single-cell behaviors play essential roles during early-stage biofilms formation. In this study, we

18 evaluated whether biofilm formation could be guided by precisely manipulating single cells

19 behaviors. Thus, we established an illumination method to precisely manipulate the type IV pili

20 (TFP) mediated motility and microcolony formation of Pseudomonas aeruginosa by using a

21 combination of a high-throughput bacterial tracking algorithm, optogenetic manipulation and

22 adaptive microscopy. We termed this method as Adaptive Tracking Illumination (ATI). We

23 reported that ATI enables the precise manipulation of TFP mediated motility and microcolony

24 formation during biofilm formation by manipulating bis-( $\left.3^{\prime}-5^{\prime}\right)$-cyclic dimeric guanosine

25 monophosphate (c-di-GMP) levels in single cells. Moreover, we showed that the spatial

26 organization of single cells in mature biofilms can be controlled using ATI. Thus, the established

27 method (i.e., ATI) can markedly promote ongoing studies of biofilms. 


\section{Introduction}

Biofilm is the most successful lifestyle that allows microbes to survive and thrive in nature (H. C.

Flemming et al., 2016; Hall-Stoodley, Costerton, \& Stoodley, 2004; Hibbing, Fuqua, Parsek, \&

Peterson, 2010). Biofilm formation has been reported to occur in several stages; namely, the and, ultimately, the release of dispersal cells to restart the lifecycle (McDougald, Rice, Barraud,

Steinberg, \& Kjelleberg, 2012). Recent studies have indicated that single-cell motility mechanisms (Gibiansky et al., 2010), including type IV pili (TFP)- or flagella-mediated motility (Conrad et al., 2011), and EPS production (Zhao et al., 2013) play essential roles in determining the location and time of microcolony formation. These fundamental findings not only provide researchers with an excellent opportunity for developing novel strategies to prevent biofilm formation on medical and industrial settings, which can cause antibiotic-tolerant infections (Costerton, Stewart, \& Greenberg, 1999) and the destruction of flow systems, but also motivate researchers to determine whether biofilm formation can be completely controlled by precisely manipulating single-cell motility and microcolony formation during early-stage biofilm formation. Alkema, \& Samuel, 2011) and adaptive microscopy (Tischer, Hilsenstein, Hanson, \& Pepperkok, 2014) enables the direct manipulation of free-moving nematode Caenorhabditis elegans, thus motivating the development of a method that can directly manipulate many moving bacteria on a surface during biofilm formation. Similarly, in this study, using a combination of a highthroughput bacterial tracking algorithm, optogenetic manipulation, and adaptive microscopy, we 
51 first established a method, adaptive tracking illumination (ATI). This method could precisely

52 illuminate single moving cells through the in-situ analysis and tracking of moving bacterial

53 trajectories. Subsequently, we applied this method to directly manipulate the TFP-mediated

54 motility and microcolony formation of single Pseudomonas aeruginosa cells during early-stage

55 biofilm formation. Bis- $\left(3^{\prime}-5^{\prime}\right)$-cyclic dimeric guanosine monophosphate (c-di-GMP) signaling

56 pathways (Hengge, 2009) are established to govern the pleiotropic behavior of bacteria, including

57 bacterial motility, EPS production, and biofilm formation. Therefore, we modified the genome of

58 P. aeruginosa by using an optogenetic tool (M. H. Ryu \& Gomelsky, 2014), which facilitated the

59 direct manipulation of the c-di-GMP level in single cells by using near-infrared light. We observed

60 that precisely manipulating the c-di-GMP level in single cells during early-stage biofilm formation

61 enabled the guiding of subsequent organization of cells in mature biofilms. Thus, the established

62 method (i.e., ATI) can markedly promote modern research on biofilms, ranging from fundamental

63 to industrial studies.

\section{Results}

\section{ATI enables precise illumination of single $P$. aeruginosa cells.}

66

67
During biofilm formation, $P$. aeruginosa cells differently deploy their TFP to mediate distinct motility, with a mobile or an immobile phenotype (Ni et al., 2016). The process involves the frequent dispersal of mobile cells on surfaces, enabling them to find a habitat that can supply sufficient nutrients. By contrast, immobile cells stall in their place to form microcolonies. These distinct TFP-mediated motility phenotypes are thereby expected to affect subsequent biofilm formation, including the organization and differentiation of cells and the structure of mature biofilms. To precisely illuminate single $P$. aeruginosa cells with distinct motility, we first acquired 
73 the bright-field images of the cells and then tracked them to obtain their trajectories in real time.

74 This real-time information was further analyzed to identify mobile and immobile cells. Thereafter,

75 information on the regions containing selected cells with the motility phenotype of interest was

76 input into a digital micromirror device to generate a mask. The mask was finally projected on these

77 selected cells through an additional objective. We termed this illumination as ATI (Figure 1a,

78 Figure 1 -figure supplement 1 and Figure 1 -figure supplement 2). Our results indicated that

79 feedback illuminations could generate projected patterns to exactly follow the cell movement

80 (Figure 1b and Video 1) or single cells divisions (Figure 1c and Video 2) in real time.

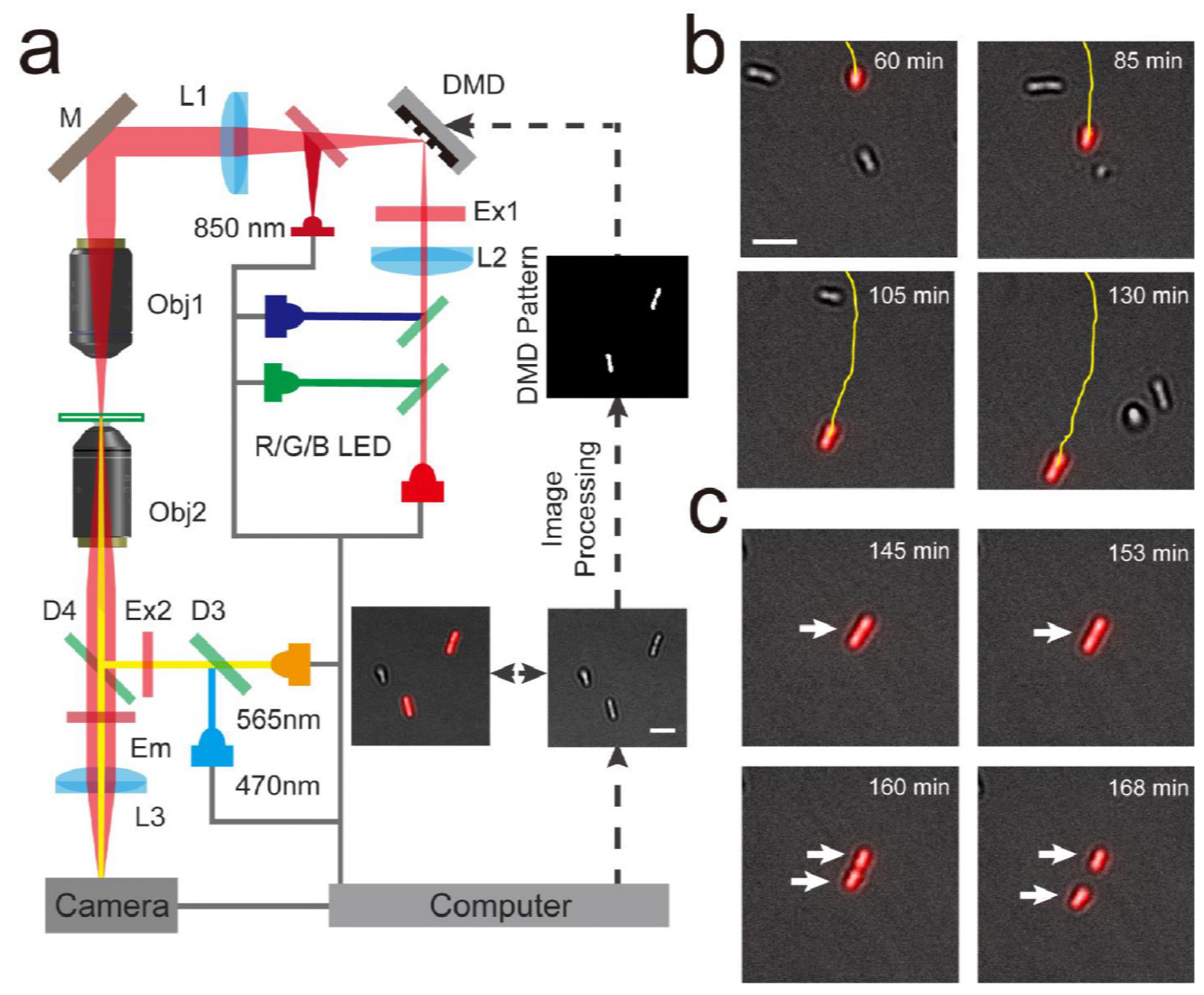




\section{Figure 1 with 2 supplements. Using Adaptive Tracking Illuminations (ATI) to exactly}

83 illuminate single $\boldsymbol{P}$. aeruginosa cells on surface. (a) Schematic drawing of the ATI system. A high-throughput bacterial tracking algorithm was employed for analyzing cells' behaviors in real time and the information were immediately feedback to an adaptive microscope equipped with a digital micromirror device (DMD). (b) One interested cell being tracked and projected in real time is depicted. (c) The feedback illuminations also can generate projected patterns to exactly follow the daughter cells after the division of one interested cell. Scale bars for all images are $5 \mu \mathrm{m}$. single cells.

To directly manipulate the TFP-meditated motility of the selected single cells, we incorporated an optogenetic part into the chromosome of $P$. aeruginosa by using the mini-CTX system(Hoang, Kutchma, Becher, \& Schweizer, 2000). This part encodes a heme oxygenase (bphO) and c-diGMP diguanylate cyclase $(b p h S)$ that can cyclize two GTP molecules to form a c-di-GMP molecule in the presence of near-infrared light (M. H. Ryu \& Gomelsky, 2014). Furthermore, the optogenetic part enabled the direct manipulation of intracellular c-di-GMP levels by using illumination at 640 $\mathrm{nm}$ (Figure 2a). Elevation of c-di-GMP levels is known to repress bacterial motility and promote biofilm formation (Hengge, 2009). To optimize the intensity of manipulation lights, we varied the LED light intensity $(640 \mathrm{~nm})$ to stimulate optogenetically modified bacteria. Furthermore, we monitored c-di-GMP levels in single cells by using a fluorescent reporter (Rybtke et al., 2012).

101 The c-di-GMP reporter encodes two fluorescent proteins. A green fluorescent protein (GFP) is expressed using a c-di-GMP regulatory promoter (PcdrA), and a red fluorescent protein (mCherry)

103 is expressed using a constitutive promoter (PA1O4O3; Figure 2a). Thus, the ratio of fluorescent intensities derived from GFP $\left(\mathrm{F}_{\mathrm{G}}\right)$ and mCherry $\left(\mathrm{F}_{\mathrm{R}}\right)$ was used to determine the c-di-GMP levels 

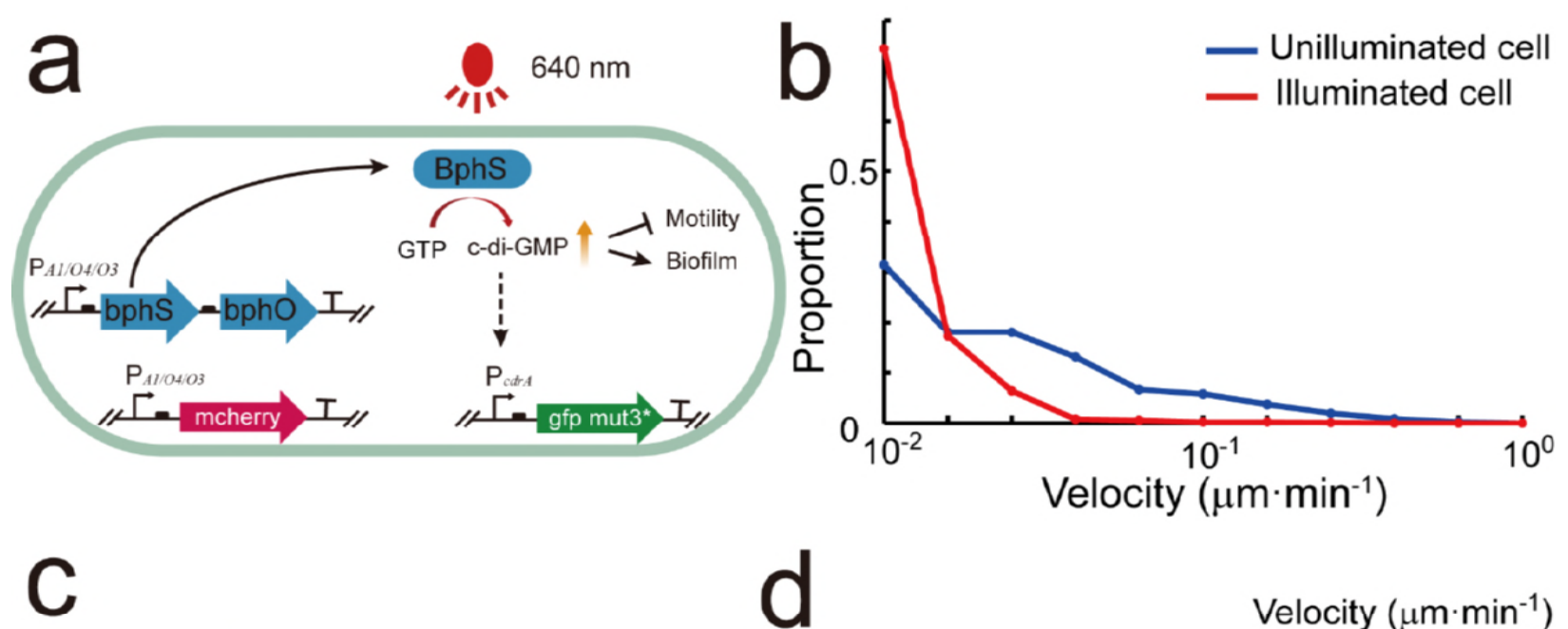

Velocity $\left(\mu \mathrm{m} \cdot \mathrm{min}^{-1}\right)$
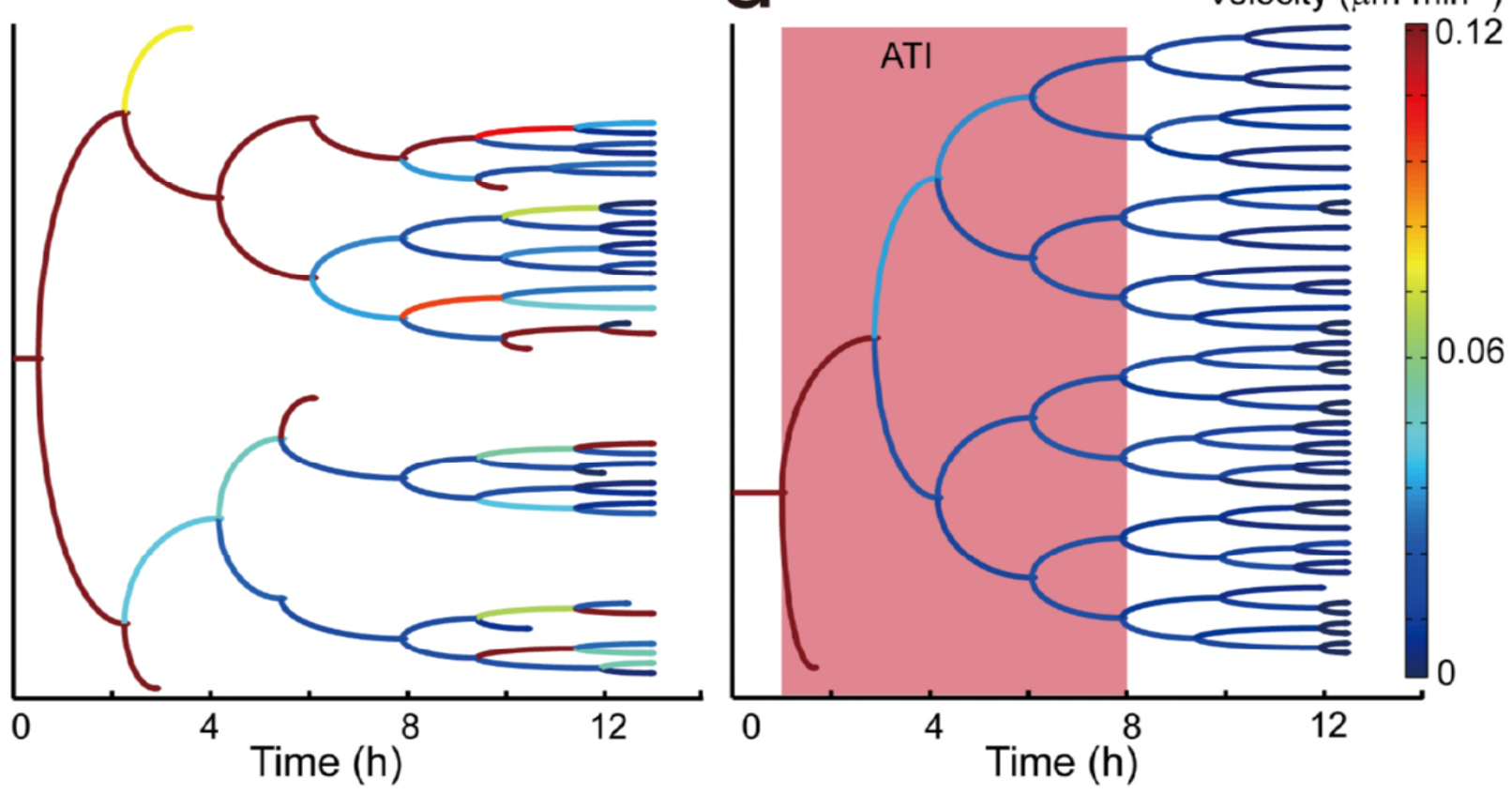

Figure 2 with 2 supplements. By introducing optogenetic modules, the ATI can precisely manipulate TFP meditated motility. (a) Schematic of optogenetic circuit. Optogenetic modules

112 encode a c-di-GMP diguanylate cyclase (BphS), which allowed us to manipulate c-di-GMP level 
113

114

115

116

117

118

119

120

121

122

123

124

125

126

127

128

129

130

131

132

133

134

135

using an illumination of $640 \mathrm{~nm}$ lights. Expression of GFP mut3* under control of the c-di-GMP regulatory promoter (PcdrA) was used for monitoring the c-di-GMP levels. A mCherry reporter fused to the constitutive promoter PA1/O4/O3 served as an internal control. (b) The illuminated cells have much slower velocity than unilluminated cells. Data were analyzed and obtained after 4 hours of manipulation by ATI. (c) Genealogical tree of one unilluminated cell and (d) one illuminated cell. The velocity of each cell is color-coded along each lineage. ATI enabled mobile cells and their offspring to switch to immobile phenotype as indicted by their averaged moving velocities decreased from 0.2 to $0.01 \mu \mathrm{m} \cdot \mathrm{min}^{-1}$ and less detachment events. Experiments in b-d were carried out at least three times and one representative example is shown.

We first selected $33-66 \%$ of single cells with mobile motility and illuminated them (0.05 $\mathrm{mW} \cdot \mathrm{cm}^{-2}$ ) along with their offspring for 7 hours by using ATI. Treatment with ATI for 3 hours enabled all mobile cells and their offspring to switch to the immobile phenotype, as indicated by their average moving velocities, which decreased from 0.2 to $0.01 \mu \mathrm{m} \cdot \mathrm{min}^{-1}$ (Figs. $2 \mathrm{~b}$ and $\mathrm{d}$ and Video 3). This observation contrasted with the observation that $50 \%$ of mobile cells $(>0.03$ $\mu \mathrm{m} \cdot \mathrm{min}^{-1}$ ) without ATI treatment remained mobile on surfaces (Figure $2 \mathrm{~b}$ and $\mathrm{c}$ and Video 3 ). Wild-type $P$. aeruginosa can spontaneously switch between mobile and immobile phenotypes during early-stage biofilm formation(Mikkelsen, Sivaneson, \& Filloux, 2011), which results in that the velocity distribution arising from single cells are broadly distributed from 0.01 to 0.5 $\mu \mathrm{m} \cdot \min ^{-1}$ (Figure $2 \mathrm{~b}$ ). Moreover, we observed that manipulation by using ATI markedly enhanced the possibility that the post-division cells remained attached to surfaces (Figure 2d). This observation contrasted with the observation that unilluminated cells exhibited asymmetrical detaching behaviors after division (Figure 2c): one daughter cell preferred to remain attached to surfaces, whereas another cell preferred to detach from surfaces. 
136 Simultaneously, we observed that 7 hours of ATI treatment considerably increased the c-di-GMP

137 levels in these illuminated cells and their offspring, as indicated by the $\mathrm{F}_{\mathrm{G}} / \mathrm{F}_{\mathrm{R}}$ ratio, which increased

138 from 1.5 to 7.5 (Figure 3a, $\mathrm{d}$ and Video 3). This observation was in sharp contrast to the observation

139 that c-di-GMP levels remained low in unilluminated mobile cells (Figure 3a, c and Video 3). The

140 mCherry-induced fluorescence remained nearly constant in the illuminated and unilluminated cells

141 (Figure 2-figure supplement 2). Notably, the optogenetic manipulation of single mobile cells and

142 their offspring enabled these cells to stall in their place to form a microcolony during biofilm

143 formation, whereas mobile cells and their offspring without optogenetic manipulation were more

144 likely to move and spread on surfaces (Figure $3 \mathrm{~b}$ and Video 3). To evaluate how optogenetic

145 manipulation affects microcolony formation, we calculated the clustering ability of illuminated

146 and unilluminated cells through hierarchical clustering. Clustering calculation indicated that ATI

147 enabled single mobile cells to form a microcolony, as indicated by the distance among the daughter

148 cells $(<4 \mu \mathrm{m}$; Figure $3 \mathrm{~d}$ and $\mathrm{f})$. This observation was in sharp contrast to the observation that

149 unilluminated cells and their offspring were more likely to spread on surfaces, as indicated by the

150 distance among these daughter cells (> $40 \mu \mathrm{m}$; Figure $3 \mathrm{c}$ and e). 

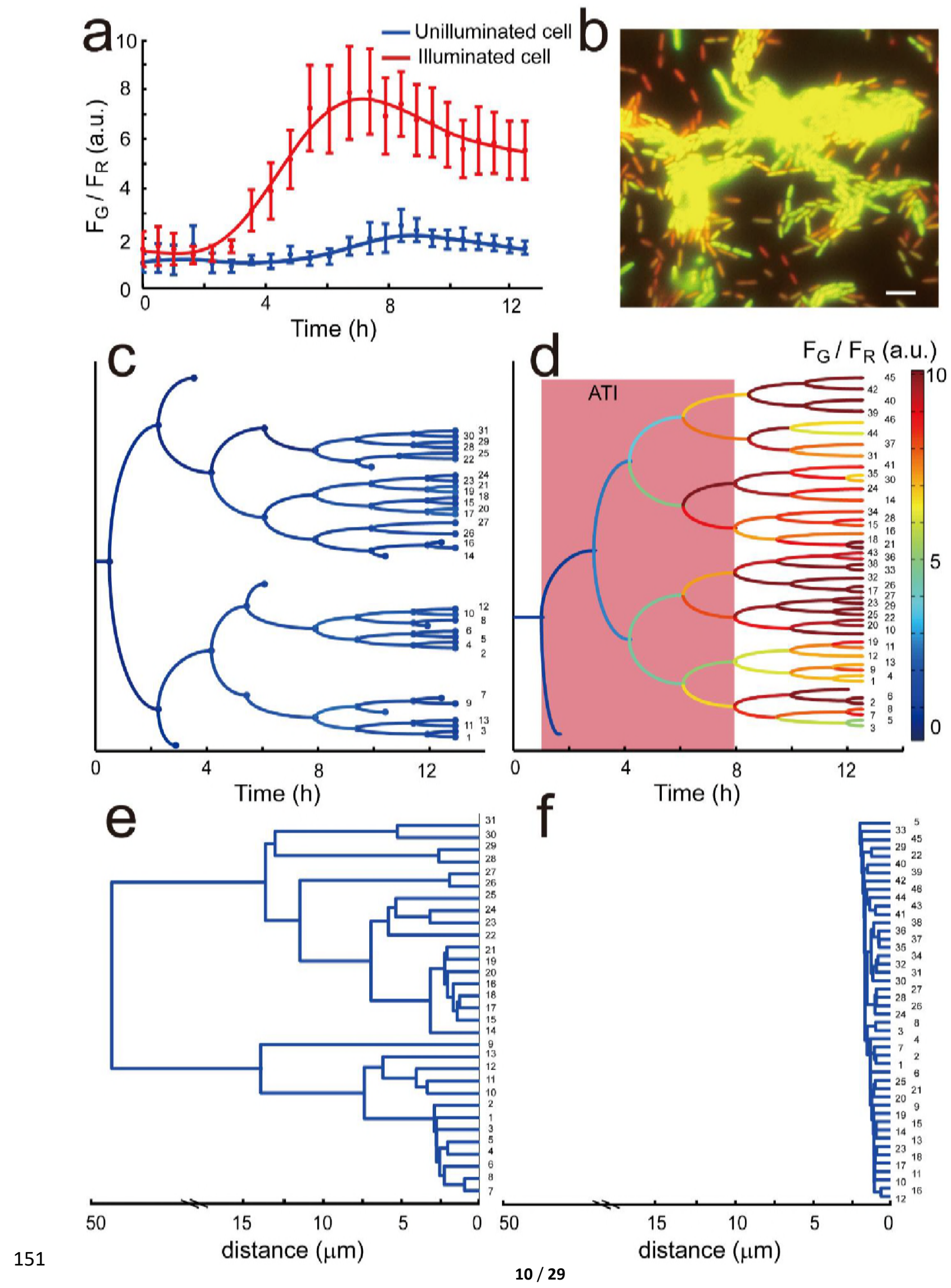

$F_{G} / F_{R}$ (a.u.) 
152 Figure 3. c-di-GMP level was manipulated using ATI system in single cells. (a) The

153 illuminated cells and their offspring have fivefold higher levels of c-di-GMP than unilluminated 154 cells after 6 hours as indicated by the contrast of $F_{G} / F_{R}$. Error bars represent means $\pm s$. $d$ with $n=$

1553 biological replicates. (b) A merged image of mCherry and GFP mut3* fluorescence microscopy

156 image was presented after 14 hours. The illuminated cells have brighter green fluorescence, which

157 indicates higher c-di-GMP level. Scale bar were set at $5 \mu \mathrm{m}$. The c-di-GMP level from one mother

158 cell is depicted by Genealogical trees for unilluminated (c) and illuminated cell (d), respectively.

159 (e) and (f) for the corresponding hierarchical clustering analysis in (c) and (d). After manipulation

160

161

162

163

by ATI for 12 hours, the distance among the daughter cells was less than $4 \mu \mathrm{m}$, which is in clear contrast to the largest distance between unilluminated daughter cells larger than $40 \mu \mathrm{m}$. Experiments in b-f were carried out at least three times and one representative sample is shown.

\section{ATI enables the guiding of biofilm formation in $P$. aeruginosa.}

After 7 hours of illumination, c-di-GMP levels remained high in the cells and their offspring, even without further optogenetic manipulation (Video 3). This behavior guided subsequent biofilm organization by manipulating microcolony formation during early-stage biofilm formation. To demonstrate this phenomenon, we labeled the optogenetically modified cells by using a green or red fluorescent protein (EGFP or mCherry), which did not contain the fluorescent reporter of c-diGMP. We cocultured these differently labeled cells in a flow cell to facilitate their biofilm formation, during which, we only selected GFP- or RFP-labeled cells and manipulated them by using ATI in the first 10 hours. Our results indicated that ATI enabled the selected cells (GFP- or RFP-labeled cells) and their offspring to form microcolonies in advance, whereas the unselected cells distributed around the formed microcolonies (Figure 4a). This result was in sharp contrast to that obtained for GFP- and RFP-labeled cells without ATI treatment (Figure 4-figure supplement 
bioRxiv preprint doi: https://doi.org/10.1101/229229; this version posted December 4, 2017. The copyright holder for this preprint (which was not certified by peer review) is the author/funder, who has granted bioRxiv a license to display the preprint in perpetuity. It is made available under aCC-BY 4.0 International license.

175 1). Thereafter, we continuously cultured these young biofilms (up to 72 hours) with a distinct 176 organization of cells in dark to allow their maturation. These distinct young biofilms developed to 177 mature biofilms possessing a distinct spatial organization of GFP- or RFP-labeled cells (Figure 178 4b). These results revealed that cell manipulation by using ATI during early-stage biofilm 179 formation enabled the guiding of biofilm formation.
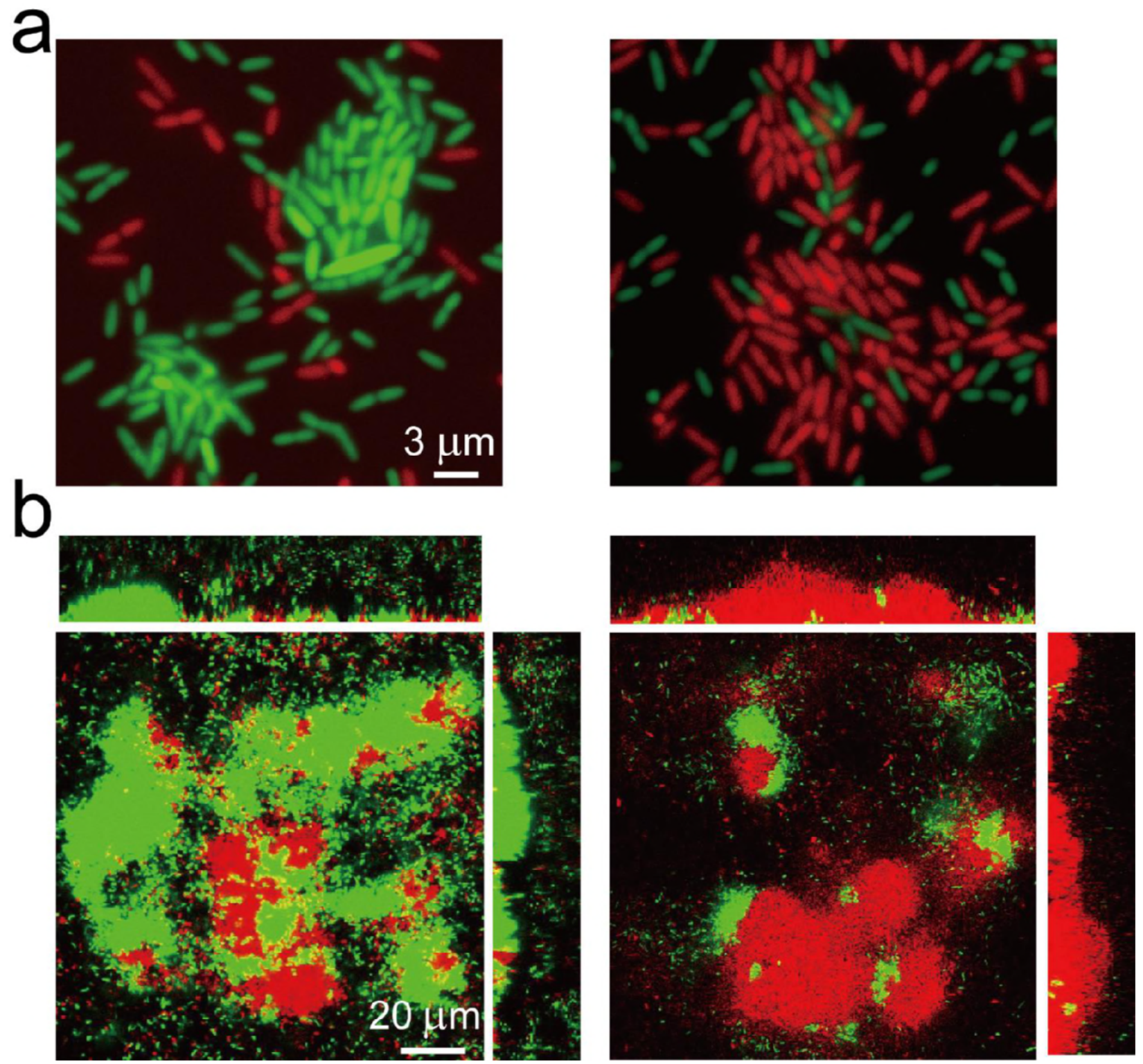
Figure 4 with 1 supplements. Guiding biofilm formation using ATI in P. aeruginosa. (a) ATI enables selected cells and their offspring to form microcolonies in advance. The optogenetic modified cells (do not contain fluorescent reporter of c-di-GMP) labeled by a green (EGFP) or red (mCherry) fluorescent protein were used for biofilm cultivation in flow cell. Left panel, EGFPlabeled cells were manipulated by ATI; Right panel, mCherry-labeled cells were selected for manipulation. The fluorescence image was attained in flow cell at the same time point about $\mathrm{t} \sim 14$ the RFP-labeled cells possess distinctive spatial organizations of biofilms. Experiments in a-b were carried out at least three times and one representative image is depicted.

\section{Discussion}

191 We developed the ATI method that could be used to precisely manipulate TFP-mediated motility 192 of single $P$. aeruginosa cells during early-stage biofilm formation. In this method, the bacteria 193 were modified using an optogenetic part that enabled illumination with near-infrared light to 194 directly regulate intracellular c-di-GMP levels. We showed that ATI could manipulate single cells 195 with a mobile phenotype to switch to an immobile phenotype. Consequently, these manipulated cells could stall in their place to form microcolonies in advance, whereas unmanipulated cells with

197 a mobile phenotype were more likely to move and spread on surfaces, facilitating the control of 198 the location and time of early-stage biofilm formation. Accordingly, we showed that the spatial 199 organization of single cells could be precisely controlled in a young biofilm of $P$. aeruginosa cells.

200 Notably, our results indicated that the organization of single cells in young biofilms affected 201 subsequent cell organization in mature biofilms, which enabled the further control of the structure and spatial organization of cells in biofilms. It should be emphasized that the strategy used herein 
to control the spatial organization of bacterial colonies is different than that using micro-threedimensional printing (Connell, Kim, Shear, Bard, \& Whiteley, 2014).

In addition to using ATI to guide biofilm formation, we expect that our method can be used to answer various questions or resolve problems in microbiology. This is because: 1) The hardware used to build ATI, mainly an air objective, a commercial projector, and an LED controller, are quite common and inexpensive; 2) The optical setup of ATI is compatible with that of other commonly used microscopic techniques, including fluorescence, confocal, and total internal reflection microscopy; 3) The wavelengths in ATI can be easily expanded to multiple colors to adapt to different optogenetic tools (Ohlendorf, Vidavski, Eldar, Moffat, \& Moglich, 2012; Olson, Hartsough, Landry, Shroff, \& Tabor, 2014; M.-H. Ryu, Fomicheva, Moskvin, \& Gomelsky, 2017); and 4) The software and algorithms used in ATI, including image processing, single-cell tracking, and analysis of phenotypes, are quite flexible to modifications. These factors allow researchers to simply integrate the ATI setup to their microscope and quickly modify the algorithms to track single cells with phenotypes of their interest, thus markedly prompting studies of optogenetic tools. Notably, the time required for data processing limits the application of ATI for investigating a quickly evolving bio-systems or cellular processes. For example, data processing of a live image (processed in a commercial desktop equipped with an intel i7 CPU) in the present study typically took 3 seconds, limiting the use of ATI for manipulating rapidly swimming bacteria, whose velocity can typically reach tens of microns per second. In addition to the data processing speed, the accuracy of the tracking algorithm limits the application of ATI for investigating microbes with high cell densities. For example, the current ATI cannot be used to manipulate the phenotypes of single cells during middle-stage biofilm formation because the algorithm cannot accurately 
225

226

227

228

229

230

231

232

track single cells in a dense microcolony. Developing new tracking algorithms or using powerful computers can address these limitations, thus considerably expanding ATI applications.

\section{Materials and Methods}

\section{Strains and Growth conditions}

Bacterial strains and plasmids used in this study are listed in Table 1. Strains were grown on LB agar plates at $37^{\circ} \mathrm{C}$ for 24 hours. Monoclonal colonies were inoculated and cultured with a minimal medium $(\mathrm{FAB})$ at $37^{\circ} \mathrm{C}$ with $30 \mathrm{mM}$ glutamate as carbon source under an aerobic condition, in which the medium contains following compositions per liter: $\left(\mathrm{NH}_{4}\right)_{2} \mathrm{SO}_{4}, 2 \mathrm{~g} / \mathrm{L} ; \mathrm{Na}_{2} \mathrm{HPO}_{4} \cdot 12 \mathrm{H}_{2} \mathrm{O}$, $12.02 \mathrm{~g} / \mathrm{L} ; \mathrm{KH}_{2} \mathrm{PO}_{4}, 3 \mathrm{~g} / \mathrm{L} ; \mathrm{NaCl}, 3 \mathrm{~g} / \mathrm{L} ; \mathrm{MgCl}_{2}, 93 \mathrm{~g} / \mathrm{mL} ; \mathrm{CaCl}_{2} \cdot 2 \mathrm{H}_{2} \mathrm{O}, 14 \mathrm{~g} / \mathrm{mL} ; \mathrm{FeCl} 3,1 \mathrm{uM}$; and trace metals solution $\left(\mathrm{CaSO}_{4} \cdot 2 \mathrm{H}_{2} \mathrm{O}, 200 \mathrm{mg} / \mathrm{L} ; \mathrm{MnSO}_{4} \cdot 7 \mathrm{H}_{2} \mathrm{O}, 200 \mathrm{mg} / \mathrm{L} ; \mathrm{CuSO}_{4} .5 \mathrm{H}_{2} \mathrm{O}, 20\right.$ $\left.\mathrm{mg} / \mathrm{L} ; \mathrm{ZnSO}_{4} \cdot 7 \mathrm{H}_{2} \mathrm{O}, 20 \mathrm{mg} / \mathrm{L} ; \mathrm{CoSO}_{4} \cdot 7 \mathrm{H}_{2} \mathrm{O}, 10 \mathrm{mg} / \mathrm{L} ; \mathrm{NaMoO}_{4} \cdot \mathrm{H}_{2} \mathrm{O}, 10 \mathrm{mg} / \mathrm{L} ; \mathrm{H}_{3} \mathrm{BO}_{3}, 5 \mathrm{mg} / \mathrm{L}\right)$ $1 \mathrm{~mL} / \mathrm{L}$. The strains were harvested at $\mathrm{OD}_{600}=2.1$, and the bacterial cultures were further diluted (1:100) in fresh FAB medium to OD600 $=0.4 \sim 0.6$ before used.

Table 1. Strains, plasmids, and primers used in this study.

\begin{tabular}{|lll|}
\hline Categories & Description & Source \\
\hline Strains & & \\
PAO1 & Wild type P.aeruginosa strain & PMID: \\
& & \\
PAO1- $b p h S$ & $b p h S$ derivative of PAO1 (wild type) & This study \\
PAO1- $b p h S$-mCherry & $b p h S$ derivative of PAO1 marked with mCherry & This study \\
PAO1- $b p h S$-EGFP & $b p h S$ derivative of PAO1 marked with EGFP & This study \\
PAO1- $b p h S$-mCherry- & $b p h S$ derivative of PAO1 marked with mCherry carrying with & This study \\
pUCP22-PcdrA-GFP & a c-di-GMP reporter plasmid based on pUCP22 & \\
\hline Plasmids & &
\end{tabular}




\begin{tabular}{|c|c|c|}
\hline $\operatorname{mini}-\mathrm{CTX} 2-b p h S$ & $\begin{array}{l}\mathrm{Tc}^{\mathrm{r}} \text {; insertion of } b p h S \text { with } \mathrm{PA} 1 / O 4 / O 3 \text { promoter constructed } \\
\text { by PCR and cloned to HindIII/SpeI sites of mini-CTX2 }\end{array}$ & This study \\
\hline mini-Tn7-mCherry & $\begin{array}{l}\mathrm{Ap}^{\mathrm{r}}, \mathrm{Gm}^{\mathrm{r}} \text {; insertion of mCherry with } P A 1 / O 4 / O 3 \text { promoter } \\
\text { constructed by PCR and cloned to KpnI/HindIII sites of mini- } \\
\text { Tn7 }\end{array}$ & This study \\
\hline mini-Tn7-EGFP & $\begin{array}{l}\mathrm{Ap}^{\mathrm{r}}, \mathrm{Gm}^{\mathrm{r}} \text {; insertion of EGFP with } P A 1 / O 4 / O 3 \text { promoter } \\
\text { constructed by PCR and cloned to KpnI/HindIII sites of mini- } \\
\mathrm{Tn} 7\end{array}$ & This study \\
\hline pUCP22-PcdrA-GFP & $\mathrm{Gm}^{\mathrm{r}}$; c-di-GMP reporter plasmid based on pUCP22 & $\begin{array}{l}\text { PMID: } \\
22582064\end{array}$ \\
\hline pTNS2 & $\begin{array}{l}\text { Ap }{ }^{\text {r}} \text {; a helper plasmid for mini-Tn7 site-specific transposition } \\
\text { system }\end{array}$ & $\begin{array}{l}\text { PMID: } \\
15908923\end{array}$ \\
\hline pIND4 & plasmid carrying the $b p h S$ gene & $\begin{array}{l}\text { PMID: } \\
19684165\end{array}$ \\
\hline mini-CTX2 & Tet $^{r}$; tet-FRT-attP-MCS, ori, int, and oriT & $\begin{array}{l}\text { PMID: } \\
10610820\end{array}$ \\
\hline $\begin{array}{l}\text { pUC18T-mini-Tn7T- } \\
\text { Gm }\end{array}$ & $\mathrm{Ap}^{r}, \mathrm{Gem}^{r} ;$ on mini-Tn7T; oriT on pUC18 & $\begin{array}{l}\text { PMID: } \\
15908923\end{array}$ \\
\hline pFlp2 & $\mathrm{Ap}^{r}$, source of Flp recombinase & $\begin{array}{l}\text { PMID: } \\
9661666\end{array}$ \\
\hline \multicolumn{3}{|l|}{ Primers Sequence } \\
\hline$b p h S$-HindIII-For & 5'-GCCAAGCTTATGGCTAGAGGGTGCCTCAT-3' & This study \\
\hline$b p h S$-SpeI-Rev & 5'-GCCACTAGTTCCTTCATACCCGCCGGG-3' & This study \\
\hline mCherry-KpnI-For & 5'-CGGGGTACCTGCCACCTGACGTCTAAGAA-3' & This study \\
\hline mCherry-HindIII-Rev & 5'-GAGATAAGCTTTTACTTGTACAGCTCGTCCATG-3' & This study \\
\hline EGFP-KpnI-For & 5'-CGGGGTACCTGCCACCTGACGTCTAAGAA-3' & This study \\
\hline EGFP-HindIII-Rev & 5'-GAGATAAGCTTTTACTTGTACAGCTCGTCCATG-3' & This study \\
\hline
\end{tabular}

Construction of optogenetics and c-di-GMP reporter strain in $P$. aeruginosa.

240 Insertion mutant $b p h S$ was constructed by mini-CTX system using a modified procedure for

241 P.aeruginosa. The bphS mutants marked with different fluorescent proteins were constructed by

242 mini-Tn7 site-specific transposition system using a modified procedures for P.aeruginosa. We

243 constructed unmarked insertion mutants by Flp-mediated excision of the antibiotic resistance 
marker. Firstly, $b p h S$ fragments obtained from the plasmid pIND4 was cloned into the vector miniCTX2 with the PA1/O4/O3 promoter in the upstream of MCS via a two-piece ligation. The constructed plasmid was electroporated into PAO1 and the corresponding recombinant strain was identified by screening on LB agar plates containing $1 \mathrm{mM}$ IPTG and $100 \mu \mathrm{g} \cdot \mathrm{mL}^{-1}$ tetracycline. Thereafter, the strains were electroporated with a pFLP2 plasmid and distinguished on LB agar plates containing $5 \%(\mathrm{w} / \mathrm{v})$ sucrose for the excision of resistance marker. Further, the $b p h S$ mutants were marked with mCherry/EGFP by using mini-Tn7 system as the similar procedure with miniCTX. C-di-GMP reporter plasmid was electroporated to the mCherry/EGFP marked $b p h S$ mutants to determine the intercellular c-di-GMP level as required. The constructed plasmids and strains are listed in the Table 1.

\section{Setup of Adaptive Tracking Illuminations}

We schematically show the setup of the Adaptive Tracking Illuminations (ATI) (Figure 1a). More specifically, an inverted fluorescent microscope (Olympus, IX71) was modified to build the ATI. The modification includes that: 1) A commercial DMD-based LED projector (Gimi Z3) was used to replace the original bright-field light source, in which the original lens in the projector were removed and the three-colors (RGB) LEDs were rewired to connect to an external LED driver (ThorLabs) controlled by a single chip microcomputer (Arduino UNO r3); 2) The original brightfield condenser was replaced with an air objective (40×, NA $=0.6$, Leica); and 3) an additional $850 \mathrm{~nm}$ LED light (ThorLabs) was coupled to the illumination optical path using a dichroic mirror (Semrock) for the bright-field illuminations. Note that $850 \mathrm{~nm}$ LED light is a safe light to ensure that bright-field illuminations do not affect the optogenetics manipulation. The inverted fluorescent microscope equipped with a $100 \times$ oil objective and a sCMOS camera (Zyla 4.2 Andor) was used to collect bright-field images with 0.2 frame rate. The bright-field images were further 
analyzed to track multiple single cells in real time using a high-throughput bacterial tracking algorithm coded by Matlab. The projected contours of selected single cells were sent to the DMD $(1280 \times 760$ pixels $)$ that directly controlled by a commercial desktop through a VGA port. The manipulation lights were generated by the red-color LED $(640 \mathrm{~nm})$, and were projected on the

271 single selected cells in real time through the DMD, a multi-band pass filter (446/532/646, Semrock)

272 and the air objective. Figure 1-figure supplement 2 displays a demo pattern that was projected 273 using our setup, which indicate that the spatial resolution or the contrast of the micro-projection 274 reaches $1.0 \mu \mathrm{m}$ or $50: 1$, respectively.

\section{Manipulation of TFP meditated motility of single cells} Technical University) and continuously cultured at $26.0 \pm 0.1^{\circ} \mathrm{C}$ by flowing $\mathrm{FAB}$ medium

$278\left(3.0 \mathrm{~mL} \cdot \mathrm{h}^{-1}\right)$, in which the flow cell was modified by punching a hole with a $5 \mathrm{~mm}$ diameter on the channel, and the hole was sealed by a coverslip that allows the manipulation lights to pass through (Figure 1-figure supplement 2b). The inverted fluorescent microscope equipped with a $100 \times$ oil objective and a sCMOS camera was used to collect bright-field or fluorescent images with 0.2 or $1 / 1800$ frame rate respectively. The power density of manipulation lights was determined by measuring the power at outlet of the air objective using a power meter (Newport 842-PE). GFP or mCherry was excited using a $480 \mathrm{~nm}$ or $565 \mathrm{~nm}$ LED lights (ThorLabs) and imaged using single-band emission filters (Semrock): GFP (520/28 nm) or mCherry (631/36 nm). In our optogenetics manipulation experiments, the motility of single cells were continuously monitored in the absence of optogenetics manipulation using bright-field images in the first hour mobile or immobile TFP meditated motility type. The moving velocity of single cells are directly 
290 calculated by $|\mathbf{r}(t+\Delta t)-\mathbf{r}(t)| / \Delta t$, where $\mathbf{r}(t)$ is the position of the bacterium at the time $t$,

$291 \Delta t=30 \mathrm{~min}$. The cells with moving velocity larger than $0.03 \mu \mathrm{m} \cdot \mathrm{min}^{-1}$ were defined to the

292 mobile types. Afterwards, 33-66 \% mobile cells were selected to be manipulated using ATI with

293 the illumination at $0.05 \mathrm{~mW} \cdot \mathrm{cm}^{-2}$, which allowed us to compare the results arising from

294 illuminated or un-illuminated mobile cells in one experiment. The c-di-GMP levels in single cells

295 were gauged using the ratio of GFP and mCherry intensities.

\section{Guiding biofilm formation using ATI}

297 Bacterial strains of PAO1-bphS-EGFP and PAO1-bphS-mCherry were 1:1 mixed and inoculated 298 into the modified flow cell to continuously culture at $26.0 \pm 0.1^{\circ} \mathrm{C}$ by flowing $\mathrm{FAB}$ medium

$299\left(3.0 \mathrm{~mL} \cdot \mathrm{h}^{-1}\right)$. The inverted fluorescent microscope equipped with a $100 \times$ oil objective and a 300 sCMOS camera was used to collect bright-field or fluorescent images with 0.2 or 1/1800 frame 301 rate respectively. The cells with green or red fluorescence were selected respectively to be 302 manipulated using ATI with a power density of $0.05 \mathrm{~mW} \cdot \mathrm{cm}^{-2}$ during the first 10 hours. 303 Afterwards, the flow cell contains the distinctive young biofilms were continuously cultured up to 3043 days in the dark to allow these young biofilms to mature. Finally, a laser-scan confocal 305 microscope (Olympus FV1000) equipped with a 100×oil objective was used to image the cells 306 organizations as well as the three-dimensional (3D) structures of the mature biofilms using $\mathrm{z}$-axis 307 scanning $(0.5 \mu \mathrm{m}$ per step). The confocal images acquired in different z-positions were used to 308 reconstruct the structure of mature biofilms using software ImageJ. Experiments of biofilm 309 cultivation were carried out at least three times.

\section{Acknowledgements}


311 We thank Dr. M. Gomelsky for kindly providing optogenetics plasmid. This work was financially

312 supported by National Natural Science Foundation of China (21474098, 21274141, 21522406)

313 and Fundamental Research Funds for Central Universities (WK2340000066, WK2030020023).

\section{Competing interests}

315 All authors declare no competing interests.

\section{$316 \quad$ Refrences}

317 Connell, J. L., Kim, J., Shear, J. B., Bard, A. J., \& Whiteley, M. (2014). Real-time monitoring of 318 quorum sensing in 3D-printed bacterial aggregates using scanning electrochemical microscopy. 319 Proceedings of the National Academy of Sciences of the United States of America, 111(51), 1825518260. doi: 10.1073/pnas.1421211111

321 Conrad, J. C., Gibiansky, M. L., Jin, F., Gordon, V. D., Motto, D. A., Mathewson, M. A., . . .

322 Wong, G. C. L. (2011). Flagella and Pili-Mediated Near-Surface Single-Cell Motility Mechanisms

323 in P. aeruginosa. Biophysical Journal, 100(7), 1608-1616. doi: 10.1016/j.bpj.2011.02.020

324 Costerton, J. W., Stewart, P. S., \& Greenberg, E. P. (1999). Bacterial biofilms: A common cause 325 of persistent infections. Science, 284(5418), 1318-1322.

326 Flemming, H.-C., \& Wingender, J. (2010). The biofilm matrix. Nature Reviews Microbiology, 8(9), $327 \quad 623-633$.

328 Flemming, H. C., Wingender, J., Szewzyk, U., Steinberg, P., Rice, S. A., \& Kjelleberg, S. (2016). 329 Biofilms: an emergent form of bacterial life. Nature Reviews Microbiology, 14(9), 563-575. doi: 
331 Gibiansky, M. L., Conrad, J. C., Jin, F., Gordon, V. D., Motto, D. A., Mathewson, M. A., . . .

332 Wong, G. C. L. (2010). Bacteria Use Type IV Pili to Walk Upright and Detach from Surfaces.

333 Science, 330(6001), 197-U150. doi: 10.1126/science.1194238

334 Hall-Stoodley, L., Costerton, J. W., \& Stoodley, P. (2004). Bacterial biofilms: From the natural 335 environment to infectious diseases. Nature Reviews Microbiology, 2(2), 95-108. doi:

$33610.1038 /$ nrmicro821

337 Hengge, R. (2009). Principles of c-di-GMP signalling in bacteria. Nature Reviews Microbiology, 338 7(4), 263-273. doi: 10.1038/nrmicro2109

339 Hibbing, M. E., Fuqua, C., Parsek, M. R., \& Peterson, S. B. (2010). Bacterial competition: 340 surviving and thriving in the microbial jungle. Nature Reviews Microbiology, 8(1), 15-25. doi:

$341 \quad 10.1038 /$ nrmicro2259

342 Hoang, T. T., Kutchma, A. J., Becher, A., \& Schweizer, H. P. (2000). Integration-Proficient

343 Plasmids for Pseudomonas aeruginosa: Site-Specific Integration and Use for Engineering of 344 Reporter and Expression Strains. Plasmid, 43(1), 59-72. doi: 345 http://dx.doi.org/10.1006/plas.1999.1441

346 Leifer, A. M., Fang-Yen, C., Gershow, M., Alkema, M. J., \& Samuel, A. D. T. (2011). Optogenetic 347 manipulation of neural activity in freely moving Caenorhabditis elegans. Nature Methods, 8(2), 348 147-U171. doi: 10.1038/nmeth. 1554

349 McDougald, D., Rice, S. A., Barraud, N., Steinberg, P. D., \& Kjelleberg, S. (2012). Should we 350 stay or should we go: mechanisms and ecological consequences for biofilm dispersal. Nature 351 Reviews Microbiology, 10(1), 39-50. doi: 10.1038/nrmicro2695 
Mikkelsen, H., Sivaneson, M., \& Filloux, A. (2011). Key two-component regulatory systems that

Ni, L., Yang, S., Zhang, R., Jin, Z., Chen, H., Conrad, J. C., \& Jin, F. (2016). Bacteria differently

15029. doi: 10.1038/npjbiofilms.2015.29

Olson, E. J., Hartsough, L. A., Landry, B. P., Shroff, R., \& Tabor, J. J. (2014). Characterizing

364 Rybtke, M. T., Borlee, B. R., Murakami, K., Irie, Y., Hentzer, M., Nielsen, T. E., . . . Tolker365 Nielsen, T. (2012). Fluorescence-Based Reporter for Gauging Cyclic Di-GMP Levels in Pseudomonas aeruginosa. Applied and Environmental Microbiology, 78(15), 5060-5069. doi: 
374 Tischer, C., Hilsenstein, V., Hanson, K., \& Pepperkok, R. (2014). Adaptive fluorescence

375 microscopy by online feedback image analysis. In J. C. Waters \& T. Wittmann (Eds.), Quantitative

376 Imaging in Cell Biology (Vol. 123, pp. 489-503). San Diego: Elsevier Academic Press Inc.

377 Zhao, K., Tseng, B. S., Beckerman, B., Jin, F., Gibiansky, M. L., Harrison, J. J., . . Wong, G. C.

378 L. (2013). Psl trails guide exploration and microcolony formation in Pseudomonas aeruginosa

379 biofilms. Nature, 497(7449), 388-+. doi: 10.1038/nature12155 


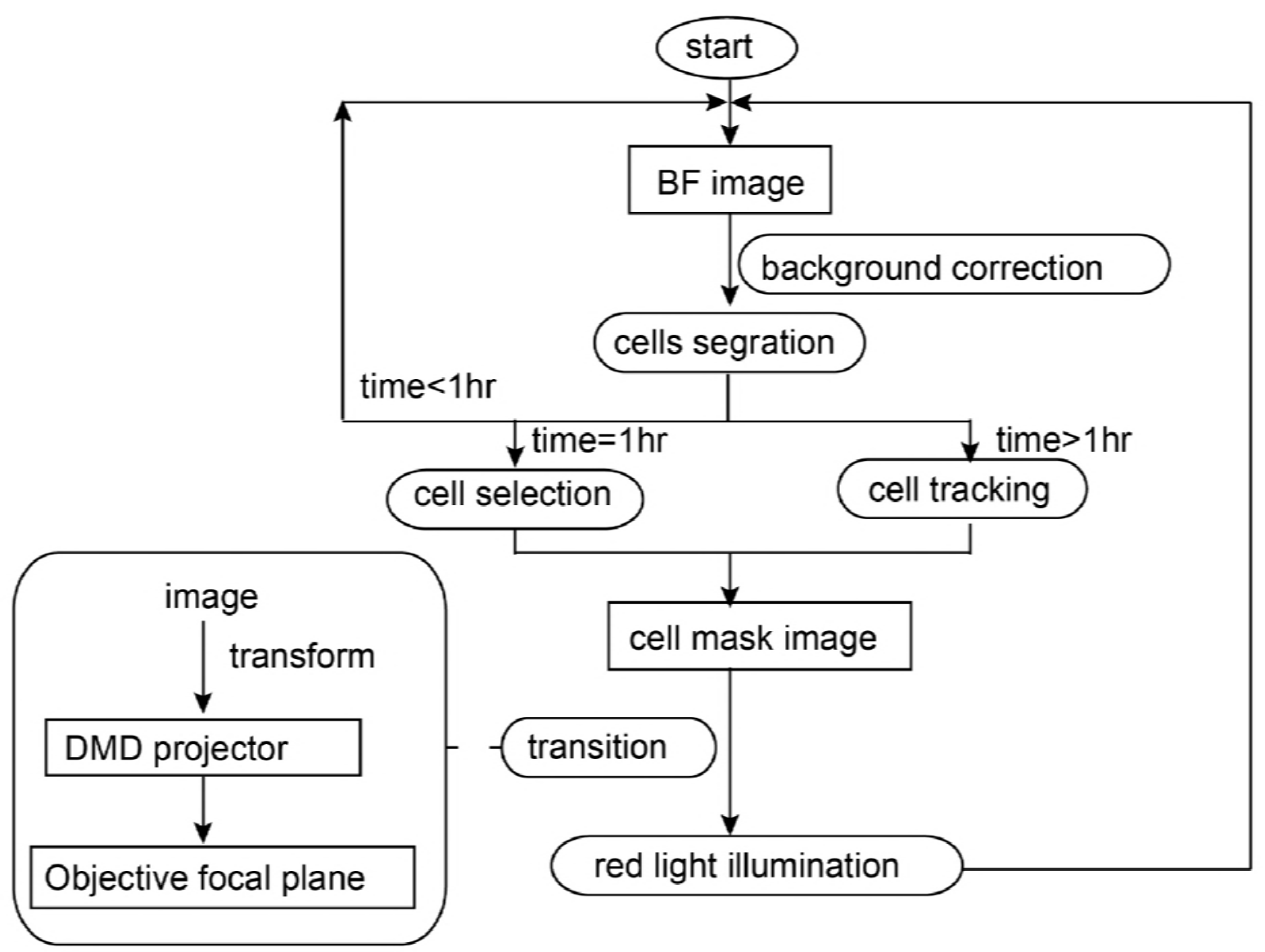

382 Figure 1-figure supplement 1. Data analysis with interactive information feedback system. The

383 process was illustrated in a simplified sequence flow diagram. Simply, bright field images were

384 taken to recognize and segment the single bacteria using an image processing algorithm coded by

385 MATLAB; Real time information of the selected cells was secondly transferred to a digital

386 micromirror device (DMD) to generate a mask; The selected cells were finally illuminated using

387 the projected mask through an additional long working distance objective. 


\section{a}

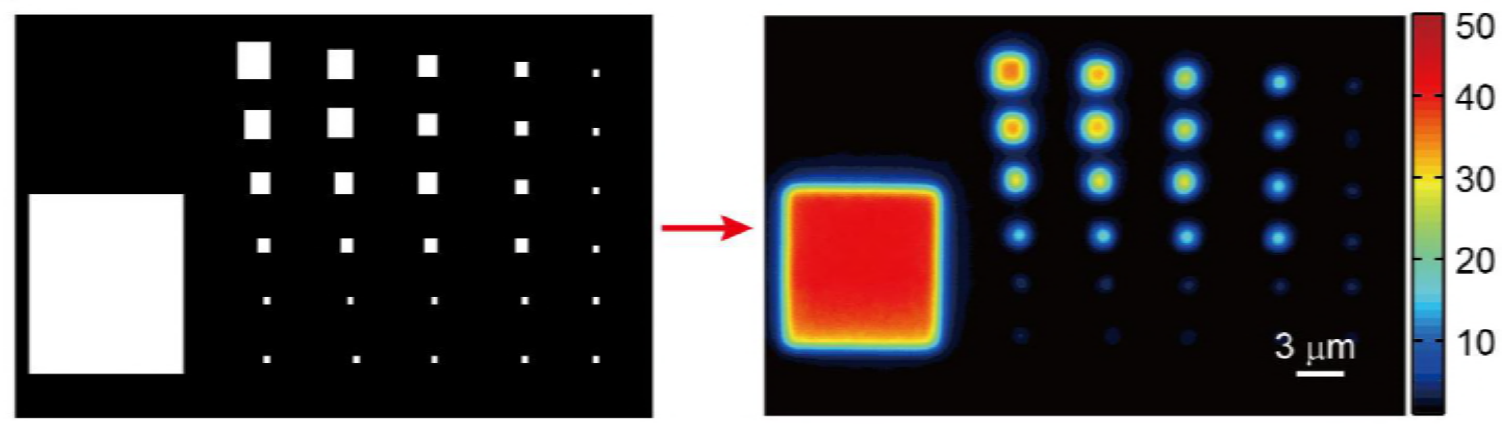

b
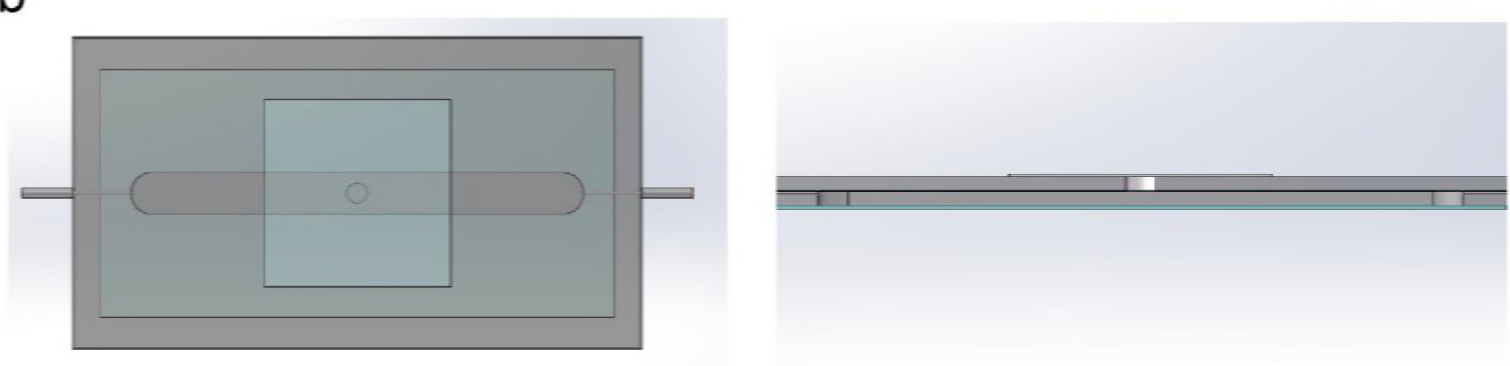

Figure 1-figure supplement 2. (a) A sequence demon patterns with different size were projected using our ATI system. The spatial resolution can reach up to $1.0 \mu \mathrm{m}$, which is smaller than length

391 of single P. aeruginosa cells (about $2 \mu \mathrm{m}$ ). The ATI system also has high contrast ratio with 50:1.

392 (b) Biofilm cultivation was carried out in an adaptive flow cell system. The schematic diagram of 393 the adaptive flow cell is presented. 

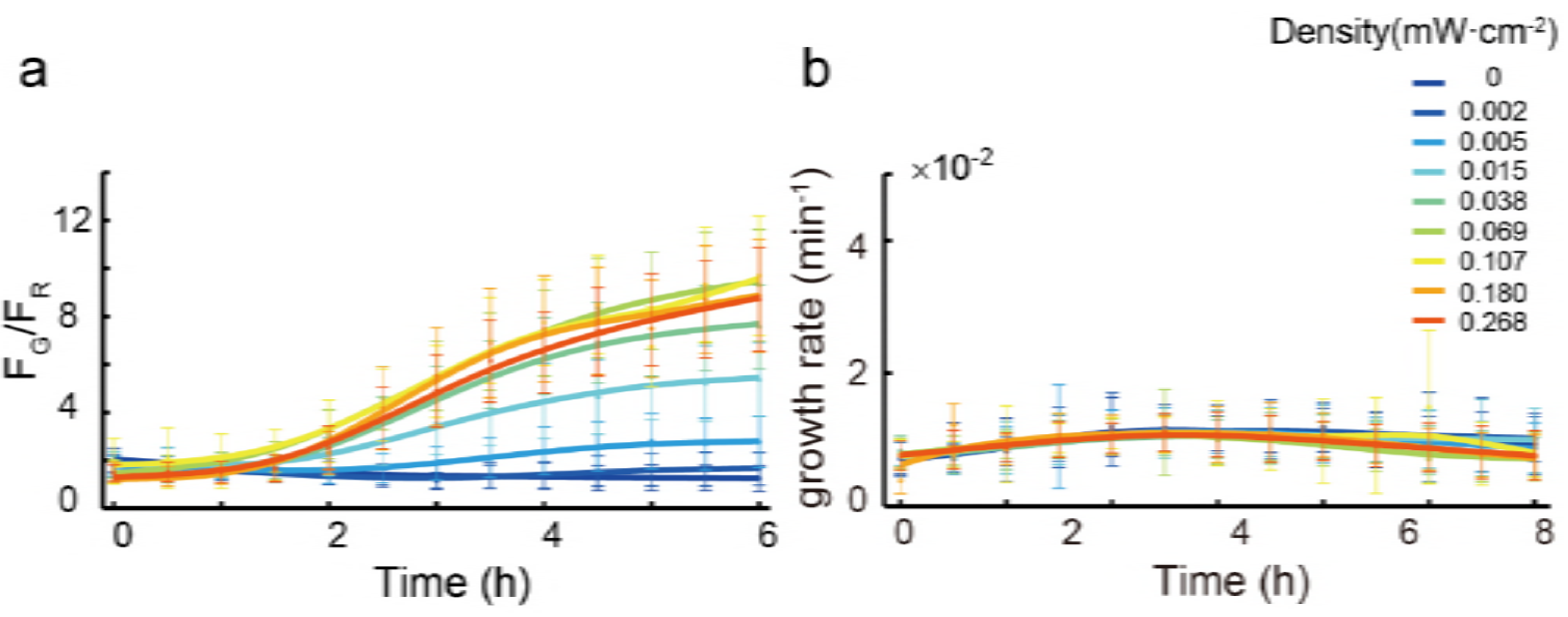

395 Figure 2-figure supplement 1. (a) Kinetics of $F_{G} / F_{R}$ were measured at indicated times with manipulation by ATI under different illumination intensity for 4 hours. (b) Relationship between

397 cell growth rate and different illuminations intensity. The intensity being chosen for carrying out experiment $\left(0.05 \mathrm{~mW} \cdot \mathrm{cm}^{-2}\right)$ does not affect the growth rate of interested cells. Error bars in a-b represent means $\pm \mathrm{s}$. $\mathrm{d}$ with $\mathrm{n}=3$ biological replicates. 

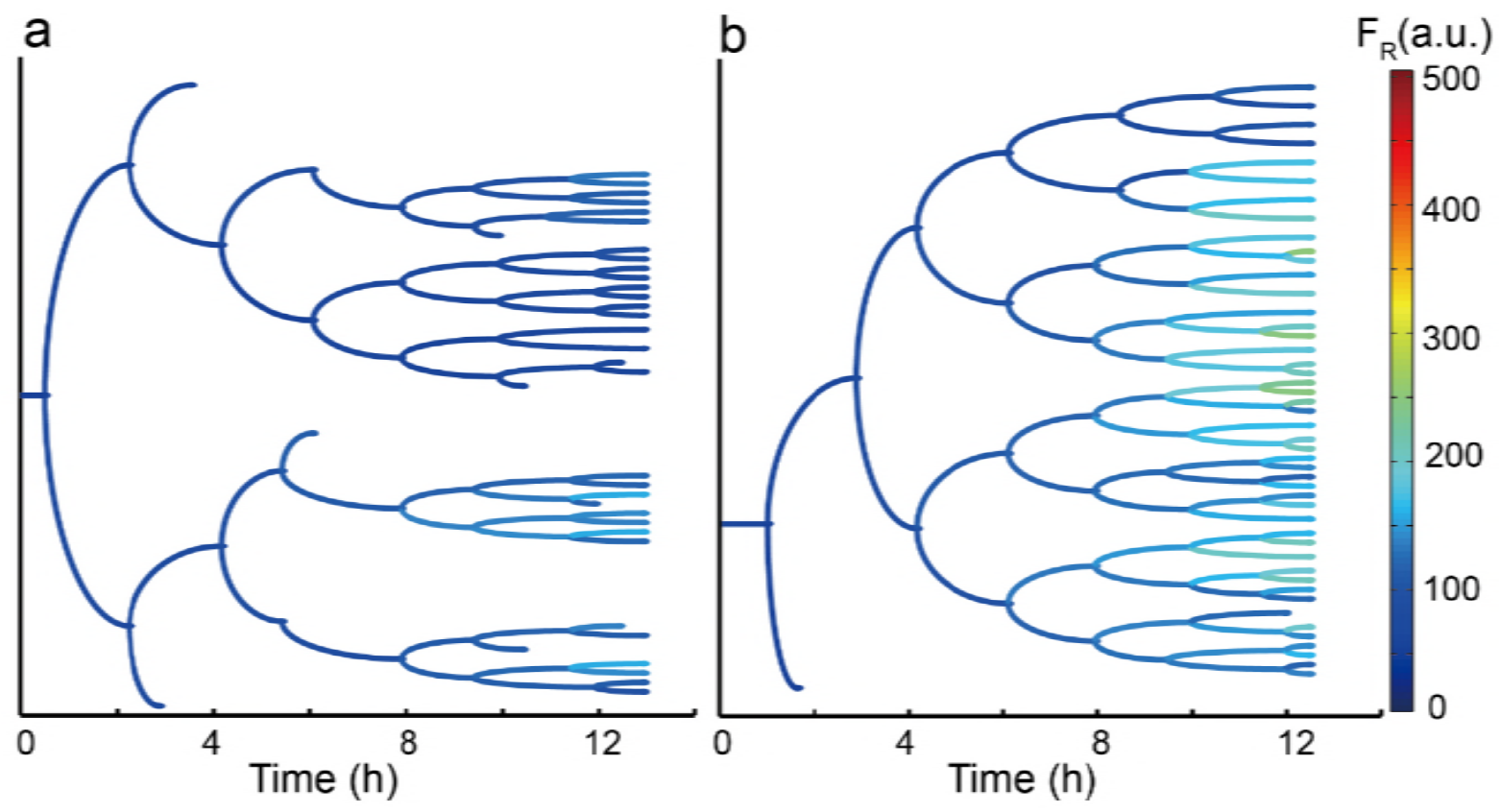

401 Figure 2-figure supplement 2. Genealogical tree of one unilluminated cell (a) and one

402 illuminated cell (b) was used to display the mCherry fluorescence intensity of its offspring 403 separately. The fluorescence arose from mCherry, which is used as an internal control, nearly 404 remains consistent in daughter cells with illuminated or unilluminated. 
a

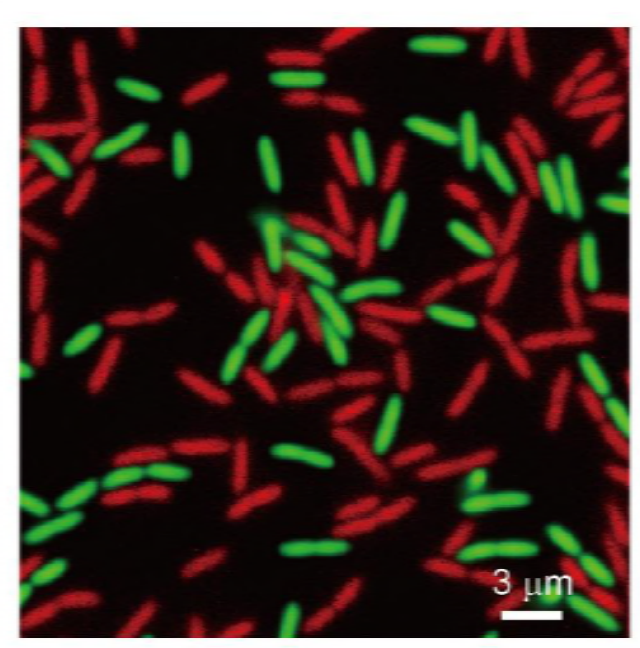

b
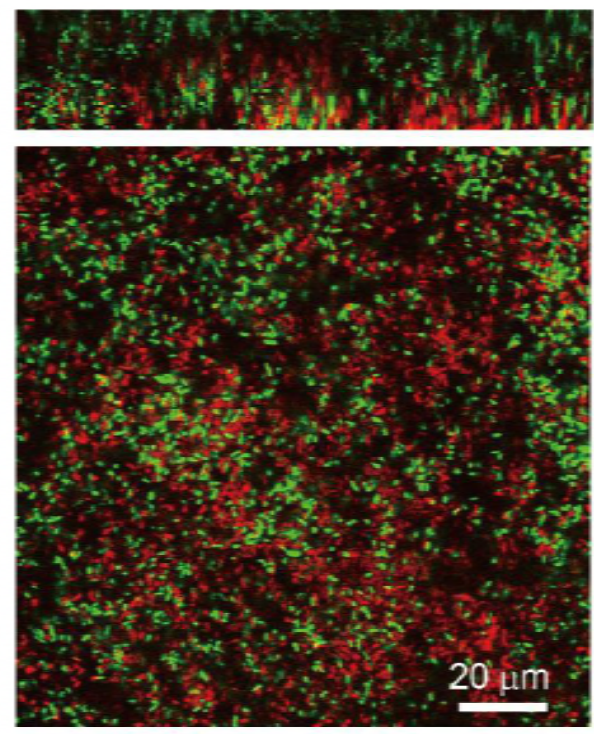

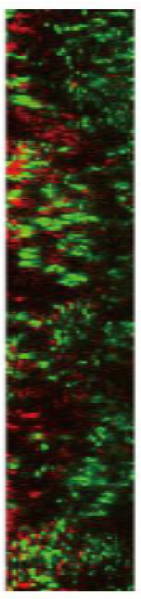

405

406 Figure 4 -figure supplement 1. Two different labeled cells with mCherry and EGFP were co-

407 cultured in a flow cell to allow them to form biofilms. No cells are selected to be manipulated by

408 ATI. (a) and (b) were captured at $\mathrm{t} \sim 10 \mathrm{~h}$ or 3 days, respectively. 
409 Video Legends

410 Video 1. One interested cell being tracked and projected in real time is depicted. Red color

411 represents the region of red LED illumination.

412 Video 2. One interested cell being tracked and projected in real time is depicted. Red color

413 represents the region of red LED illumination.

414 Video 3. Single cells are precisely illuminated by ATI via in situ analyzing and tracking bacteria.

415 The left panel shows the merged images of GFP mut3* and mCherry fluorescence microscopy

416 images changed over time. The right panel shows the merged images of red LED projected patterns

417 and bright field images as the same to left panel. The fluorescence intensity of GFP mut $3 *$ in those

418 illuminated cells and their offspring (as shown red color merged in right panel) is significantly

419 increased after using ATI for 7 hours, which is sharply contrast to that the fluorescence intensity

420 of GFP mut $3 *$ in those unilluminated mobile cells remain in low. 